\title{
Educação permanente e saúde mental: o caso da condução de grupos em Centros de Atenção Psicossocial (CAPS)
}

\section{Permanent education and mental health: the case of conducting groups in Psychosocial Care Centers (CAPS)}

\author{
${ }^{1}$ Amanda Angonese Sebben amandaangonesesmo@gmail.com \\ ${ }^{2}$ Sirlei Fávero Cetolin
}

\begin{abstract}
RESUMO
O trabalho com grupos, associado à área da saúde mental, pode superar o aspecto da normalização do cuidado com pacientes com sofrimento emocional significativo. Este artigo objetiva identificar como quatro equipes de profissionais avaliam seu trabalho na condução de grupos nos Centros de Atenção Psicossocial (CAPS), da Região de Saúde do Extremo Oeste de Santa Catarina. Realizou-se uma pesquisa qualitativa, sendo que os participantes foram doze profissionais que trabalham conduzindo grupos. Como resultados, identificou-se que o trabalho com grupos é desafiante, envolve mais pessoas e pode apresentar situações imprevistas no planejamento inicial dos profissionais. Tal fato necessariamente implica em domínio de técnicas e aprimoramento constante através da Educação Permanente para a realização das intervenções necessárias.
\end{abstract}

Palavras-chave: Trabalho em equipe. Grupo. Psicoterapia de grupo. Saúde mental. Programa de saúde mental.

\begin{abstract}
Regarding mental health, working with groups can overcome the aspect of normalization of care for patients with significant emotional distress. This article aims to identify how four teams of professionals evaluate their work in conducting groups in the Psychosocial Care Centers (CAPS) of the Region of Health of the Extreme West of Santa Catarina. A qualitative research was carried out, and the participants were twelve professionals working in groups. As a result, it has been identified that working with groups is challenging, involves more people and may present unforeseen situations in the initial planning of professionals. This fact necessarily implies in the mastery of techniques and constant improvement through Permanent Education to realize the necessary interventions.
\end{abstract}

Keywords: Team work. Group. Group psychotherapy. Mental health. Mental health program.

1 Mestre em Biociências e Saúde pela Universidade do Oeste de Santa Catarina, campus de Joaçaba. Docente do curso de graduação em Psicologia da Universidade do Oeste de Santa Catarina, campus de São Miguel do Oeste e Unidade de Pinhalzinho. Universidade do Oeste de Santa Catarina.

2 Doutora em Serviço Social pela Pontifícia Universidade Católica do Rio Grande do Sul. Docente do Programa de Pós-graduação Mestrado em Biociências e Saúde. Universidade do Oeste de Santa Catarina. 


\section{INTRODUÇÃO}

A utilização do grupo terapêutico como estratégia de assistência mostra-se importante alternativa para o trabalho em saúde mental. De acordo com o Ministério da Saúde, no trabalho com grupos associados ao campo da saúde mental deve-se evitar a formação de grupos por tipologia de sofrimento psíquico, enfatizando o grupo como um lugar de encontro entre sujeitos, buscando o aspecto comum a partir da diversidade. (SILVA, 2003; BRASIL, 2013)

No Brasil, a partir da sanção da Lei de número 10.216/2001, os direitos das pessoas portadoras de transtornos mentais passaram a ser discutidos e protegidos, redirecionando o modelo assistencial. A implantação da Política Nacional de Saúde Mental foi possível no contexto brasileiro a partir da Reforma Psiquiátrica e do Movimento Social da Luta Antimanicomial, fomentados por mobilizações dos usuários, familiares e profissionais, que lutavam desde a década de 1980 para mudar a realidade dos manicômios existentes, bem como dos serviços ofertados. (BRASIL, 2013)

Uma das propostas da Reforma Psiquiátrica é proporcionar a reinserção social e ocupacional dos usuários, possibilitando novas formas de cuidado e direcionamentos de vida. (TENÓRIO, 2002; ALVERGA; DIMENSTEIN, 2006) Conforme Barbosa, Costa e Moreno (2012), a luta do movimento é compartilhada entre usuários, familiares e profissionais, todos agentes do processo de assistência e cuidado, buscando a efetivação dos direitos e o desenvolvimento de uma maior conscientização da sociedade como um todo, procurando extinguir o preconceito e discriminação sofridos pelas pessoas com transtornos mentais.

Houve uma consequente substituição progressiva dos manicômios por outras práticas terapêuticas, sendo que a cidadania do doente mental vêm sendo objeto de discussão não só entre os profissionais de saúde, mas também em toda a sociedade. (GONÇALVES; SENA, p. 49, 2001) A partir do decreto presidencial n 7508/2011, a Rede de Atenção Psicossocial (RAPS) passa a integrar o conjunto das redes indispensáveis para as Regiões de Saúde, na oferta do cuidado aos usuários com transtornos mentais. Entre os serviços substitutivos de assistência e cuidado ao usuário portador de transtorno mental, estão os Centros de Atenção Psicossocial (CAPS). (BRASIL, 2013)

Assim sendo, pelo entendimento de que os Centros de Atenção Psicossocial são importantes serviços substitutivos das longas internações psiquiátricas e que estão presentes na região em que realizaremos o estudo, estes se caracterizaram como os cenários da pesquisa realizada. A Portaria n 336/2002 do Ministério da Saúde, produzida a partir da Lei 10.216/2011, regulamenta os Centros de Atenção Psicossocial (CAPS) que surgem com a responsabilidade da redução do número de leitos psiquiátricos, bem como para prestar atendimento clínico por meio de cuidados diários, promovendo a reinserção social de pessoas com transtornos mentais através de ações interdisciplinares. O CAPS, para tanto, conta com uma equipe multiprofissional que deve estar preparada para lidar com seu público-alvo, capacitados para disponibilizar um serviço de qualidade e intervenções direcionadas para as necessidades e demandas individuais de cada um dos usuários do serviço com transtornos mentais com comprometimento psíquico, incluindo os transtornos relacionados ao uso, abuso e dependência das substâncias psicoativas (álcool e outras drogas, lícitas ou ilícitas). (BRASIL, 2001; 2002; 2004)

Nos Centros de Atenção Psicossocial, são considerados usuários todos aqueles que apresentam um sofrimento/comprometimento psíquico intenso, que impede a realização/concretização de projetos de vida e afeta diversas esferas da vida do ser humano, seja emocional, física, social e ocupacional, incluindo os transtornos mentais e os transtornos decorrentes do uso e dependência de substâncias psicoativas. $\mathrm{O}$ acolhimento do usuário é realizado logo que o mesmo chega ao CAPS, por qualquer profissional capacitado e disponível no momento, e consiste em uma escuta do sofrimento para a identificação de sua necessidade. Após, é construído conjuntamente um plano de intervenção, individual, para atender às demandas identificadas. Caso se identifique que a pessoa não poderá ser beneficiada com o atendimento no CAPS, a mesma deverá ser encaminhada para outros serviços da rede, responsáveis pela oferta do devido cuidado. (BRASIL, 2004) 
A equipe multiprofissional pode realizar intervenções individuais sempre que estas se mostrarem como mais adequadas às necessidades dos usuários. Contudo, observa-se como a maior parte dos CAPS acaba realizando prioritariamente intervenções em grupos, sejam eles de cunho terapêutico ou operativo, conduzidos por membros da equipe multiprofissional (enfermeiros, técnicos/auxiliares de enfermagem, psicólogos, assistentes sociais, entre outros), como forma de atender às necessidades mais urgentes de seus usuários, dando conta de uma grande demanda em um curto período de tempo. (SANTOS, 2010)

O adoecimento mental é amparado por uma estrutura grupal que escora os acontecimentos individuais. Para tanto, o trabalho em grupo pode ser utilizado como forma de promover uma melhora em seus membros, já que, por intermédio da modificação dessa estrutura grupal, consegue-se a promoção da saúde psíquica de seus membros. De acordo com Castanho (2012, p. 55), "fazer com que os papéis circulem dentro do grupo, superar as estereotipias, gerar novas possibilidades de compreensão”, seriam as formas de, em um grupo, conseguir-se a promoção da saúde.

Para tanto, mostrou-se relevante investigar como os profissionais que estão inseridos nas equipes multiprofissionais destes serviços (os CAPS) percebem seus trabalhos com os usuários, quando intervém através da modalidade de grupo.

\section{MÉTODO}

Foi realizada uma pesquisa qualitativa, buscando-se conhecer o universo de significações, motivos, crenças, maneira de pensar e atitudes, alinhadas à subjetividade humana. O estudo ocorreu na Macrorregião de Saúde do Grande Oeste, mais especificamente na Região de Saúde do Extremo Oeste, com sede localizada em São Miguel do Oeste, que abrange 30 municípios. Do total, quatro municípios possuem os Centros de Atenção Psicossocial (CAPS) ativos, que são: Dionísio Cerqueira, Maravilha, Mondaí e São Miguel do Oeste.

Os doze profissionais (quatro psicólogos, três enfermeiros, dois assistentes sociais, dois arteterapeutas e um técnico de enfermagem) participantes desta pesquisa foram selecionados seguindo uma amostra não-probabilística intencional, seguindo dois critérios de inclusão: serem membros da equipe multidisciplinar dos Centros de Atenção Psicossocial (CAPS) e realizar/conduzir grupos com os usuários dos serviços, independente da característica dos grupos e de sua formação técnica/profissional. Foram realizadas entrevistas individuais por meio de perguntas estruturadas e abertas, oportunizando ao sujeito "discorrer sobre o tema proposto, sem respostas ou condições prefixadas pelo pesquisador”. (MINAYO, 2000, p. 108) As entrevistas foram transcritas para a garantia da fidedignidade das informações. Os participantes foram nomeados e numerados aleatoriamente, para proteger suas identidades.

Posteriormente, para analisar os dados coletados utilizou-se o método de análise de conteúdo, de acordo com Bardin (2016, p.31), que acredita que "a análise de conteúdo é um conjunto de técnicas de análise das comunicações”, sendo que a mesma permite explorar suas probabilidades de associações.

A pesquisa foi submetida ao Comitê de Ética em Pesquisa Humana (CEP) da Universidade do Oeste de Santa Catarina - UNOESC, de acordo com a Resolução n ${ }^{\circ}$ 466/2012, e encontra-se aprovada mediante parecer número 1.877.057 e CAAE 62849416.7.0000.5367. 


\title{
3 APRESENTAÇÃO E DISCUSSÃO DE DADOS
}

Após a análise das entrevistas realizadas com os participantes, a partir da análise de conteúdo, chegou-se a três categorias: Aspectos Positivos Sobre o Trabalho, Dificuldades Relacionadas ao Trabalho e Necessidade de Educação Permanente.

Na categoria Aspectos Positivos Sobre o Trabalho, percebeu-se como os profissionais conseguem identificar seu trabalho como satisfatório, sendo possível se surpreender a cada novo encontro. As falas dos Participantes 01, 03 e 07, ilustram a afirmativa:

\author{
“eu espero que seja maravilhoso” (Participante 01) \\ “to adorando (...) me surpreendendo a cada dia” (Participante 03) \\ "acho que esteja me esforçando assim pra estar realizando um trabalho bom (...) toda equipe, eu tam- \\ bém, me incluindo, está se esforçando para desenvolver um bom trabalho” (Participante 07)
}

A satisfação com o trabalho influencia no comportamento do profissional, bem como em sua dedicação e comprometimento com as atividades que realiza. A satisfação "pode ser compreendida como um estado emocional prazeroso que resulta de múltiplos aspectos do trabalho e que pode ser influenciada pela concepção de mundo e pelas aspirações, vivências e características individuais” (LIMA et al, 2014, p. 18), o que implica no desenvolvimento de estratégias de enfrentamento para as dificuldades que o trabalhador identifica em seu cotidiano laboral, como já discutimos anteriormente.

Além disso, o Participante 07 ressalta como a equipe também se envolve na busca pela realização eficaz do trabalho idealizado para o serviço, indicando que há um esforço coletivo. Quando o trabalho realizado pode ser compartilhado de forma colaborativa, aumenta a eficácia do atendimento, bem como a satisfação com o trabalho percebida pelo profissional. O ideal seria a constituição de um coletivo de trabalho, que superaria o trabalho em equipe, uma vez que no coletivo, todos os profissionais envolvidos buscariam formas diferentes e facilitadoras de realizar as atividades, compartilhando estratégias de intervenção e defesa. (LIMA et al, 2014; PAULA, 2011)

Foi possível verificar que há entre os participantes da pesquisa uma identificação com o trabalho em grupo, o que pode facilitar o serviço e uma avaliação positiva das características do mesmo. Quando há a identificação com aquilo que se faz, a tendência é que o trabalho seja realizado com maior qualidade, principalmente pela segurança de saber que há uma equipe dando suporte para as intervenções que serão realizadas.

"é satisfatório, eu gosto, não me vejo trabalhando em outro lugar assim, eu me encontrei” (Participante 02)

“eu gosto do que eu faço, eu acho que tá indo muito bem” (Participante 12)

O prazer encontrado com a atividade que realizam ganha destaque na fala dos profissionais ao pensar na proposta do serviço em questão: de ofertar ao usuário um atendimento holístico e único, direcionado às suas demandas e especificidades.

Mostra-se relevante que este serviço substitutivo (CAPS) apresenta ao profissional a possibilidade que ele esteja inserido no acompanhamento próximo e contínuo do usuário, enxergando-o além dos diagnósticos que carregam, percebendo os seres humanos que eles são. 
Ainda, se constatou como é satisfatório ao trabalhador acompanhar os usuários, compreendendo a influência que suas intervenções exercem na vida dessas pessoas, como podemos observar nas falas dos Participantes 02, 04 e 09:

\author{
“é bem satisfatório ver a mudança, saber da pessoa, muito bom também quando alguém tem alta por- \\ que são com pessoas com objetivo maior” (Participante 02) \\ “me vejo assim como referência deles também” (Participante 04) \\ “como o nosso trabalho é tão importante na vida dessas pessoas (...) é muito gratificante poder ajudar a \\ vida dessas pessoas (...) ver que conseguem mudar, então isso é muito gratificante né” (Participante 09)
}

Tal fato pode implicar em maior responsabilidade e comprometimento da parte do profissional, o que direciona para um serviço de maior qualidade e envolvimento. Llapa-Rodriguéz et al (2009) asseguram que o profissional engajado com o trabalho é aquele que deseja e sente que precisa permanecer realizando o que já pactuou.

Lima et al (2014) afirmam que a realização no ambiente de trabalho pode fazer a diferença na oferta de um serviço de qualidade, onde se busca a oferta do cuidado e consideração com o ser humano como prioridades. Segundo os autores, três fatores principais influenciariam na satisfação dos trabalhadores da saúde: gostar daquilo que se faz, perceber a efetividade do trabalho e identificar a realização do trabalho em equipe.

Já de acordo com a categoria Dificuldades Relacionadas ao Trabalho, conforme a autoavaliação dos profissionais dos CAPS, identificou-se como o trabalho com grupos é desafiante, principalmente ao ter que enfrentar os anseios e inseguranças profissionais ao lidar com o desconhecido ou ao confrontar-se com as crises dos usuários.

\footnotetext{
“as vezes você se frustra, porque não é como você espera que saia” (Participante 01)

“fico assim bem angustiada na questão de não poder, de não conseguir trazer mais gente” (Participante 05)

“eu acho que é um desafio que eu assumi e busco todos os dias em cumprir, tem seus altos e baixos” (Participante 08)
}

Entende-se que, como qualquer outro serviço, trabalhar com a saúde, principalmente com a saúde mental das pessoas, não se caracteriza como um trabalho fácil ou cômodo para o profissional que opta pelo mesmo. As dificuldades encontradas podem levar à insatisfação profissional, uma vez que podem refletir as condições (ou má condições) de trabalho às quais o profissional está submetido. (LIMA et al, 2014)

Percebe-se, no discurso dos profissionais, um aumento de queixas direcionadas à insatisfação com o trabalho, no sentido de não conseguir cumprir com todas as exigências preconizadas ou, pelo menos, não da maneira como estes trabalhadores gostariam. A autocobrança surge nesse contexto como uma forma da equipe demonstrar que não está sendo uma tarefa tranquila a simples execução das atividades previstas.

Esta autocobrança nunca ocorre de maneira isolada, estará interligada com sentimentos como a ansiedade e a angústia e com preocupações do sujeito em relação à insatisfação que vem sentindo. Contudo, por ela aparecer de maneira intensificada, pode demonstrar que o próprio profissional pode estar adoecendo, pois muitas vezes cobra de si algo que está além do possível, pessoalmente e institucionalmente falando. Além disso, estimula que surjam sentimentos concomitantes, como a angústia, ansiedade, insegurança e sensação de incapacidade.

A ansiedade e angústia são sentimentos associados a um sofrimento psíquico existente posterior à identificação de uma discórdia interna do sujeito (FREUD, 1915-1916). Os participantes demonstram, então, o conflito 
existente entre o que lhes era possível de ser feito e o que gostariam, no íntimo, de conseguir fazer. Paula (2011) lembra que tais sentimentos podem acarretar o adoecimento psíquico inclusive do próprio profissional, que fica em uma dicotomia entre o que é possível ser realizado e o que gostaria de realizar.

Ainda, as limitações são distinguidas pelos profissionais, ao relatarem suas dificuldades para lidar com o desconhecido e com o imprevisto, o que pode gerar insegurança na hora da condução do processo.

"no começo a gente tem aquela questão do medo do que vai acontecer, eu tenho que cuidar o que eu falo para o paciente não entender errado” (Participante 09)

"eu tenho muitas lacunas ainda, eu tenho muita coisa que eu preciso procurar ainda, aprender, estudar (...) eu tenho muito o que aprender, tem que estudar, quero fazer esses cursos de formação agora, porque eu quero ajudar eles” (Participante 12)

Souza, Vasconcellos e Amarante (2004) asseguram que o profissional que trabalha com a saúde mental deveria abrir mão da regularidade dos serviços e valorizar a imprevisibilidade de maneira a perceber que esta reflete, na maior parte das vezes, a realidade da vida da população. Salienta-se que, como o serviço se ocupa de pessoas, entende-se por natural que nem tudo saia como o planejado, principalmente considerando-se que os usuários do CAPS o frequentam justamente por apresentarem dificuldades pontuais referentes às mais variadas instâncias, com intensidade diversa. Mostra-se importante ao profissional perceber que suas intervenções devem ser planejadas, todavia, elas devem manter-se flexíveis e adaptáveis às demandas apresentadas pelos sujeitos, intervindo de acordo com as necessidades do usuário (não do trabalhador).

A terceira categoria identificada nas entrevistas com os participantes foi a respeito das Necessidades de Educação Permanente, sendo que há a preocupação entre eles quanto à qualidade do serviço, em que os participantes indicam certa falta de preparo para conduzir os grupos ao sinalizar a necessidade de maior conhecimento teórico e prático que os auxilie nesta função, como as falas dos Participantes 04 e 10 nos indicam:

\footnotetext{
"a gente precisa se apropriar mais de conhecimento pra poder passar pra eles mais segurança, mais conhecimento, adquirir para poder passar para eles” (Participante 04)
}

“eu sei que eu preciso não apenas de um conhecimento teórico, mas um preparo maior para lidar com situações de grupo (...) sinto falta de um preparo melhor” (Participante 10)

Os profissionais avaliam-se como buscando frequentemente ofertar ao usuário a melhor intervenção, sentindo a necessidade de constantemente se manterem atualizados e de adaptarem as práticas conforme as características e demandas dos usuários. Tais percepções vêm ao encontro do afirmado por Amâncio Filho (2004) em relação à impossibilidade de se pensar a atuação em saúde separada de uma formação continuada por parte do profissional. O autor acredita que o setor da saúde está em constante mudança, o que requer do profissional incessantes e inesgotáveis adequações e adaptações em sua forma de atuação.

Ainda, a equipe identifica uma insegurança quanto ao desejo de precisar saber mais, referente ao preparo teórico para lidar com as dificuldades, indicando a necessidade da Educação Permanente. Rossoni e Lampert (2004) afirmam que apenas a aquisição de conhecimentos teóricos e habilidades técnicas não são suficientes para a atuação profissional, sendo necessária a aquisição de competências sociais e relacionais, assim como de constante aprimoramento. Indo além, podemos inferir que a dificuldade da rede em entender a forma diferenciada de atuação do CAPS, pode ser decorrente da falta de preparo teórico/técnico de alguns profissionais.

Amâncio Filho (2004, p. 378) assegura a relevância da formação, qualificação, treinamento e atualização dos profissionais da saúde, pois a responsabilidade que recai sobre eles de ofertar um serviço de qualidade e 
cuidado à população é grandiosa, incitando o profissional a "integrar conhecimentos gerais e específicos, habilidades teóricas e práticas, hábitos, atitudes e valores éticos”.

Em relação à prática da Educação Permanente nos Centros de Atenção Psicossocial, podemos citar o Matriciamento, entendido como

Ação de articulação da Rede de Atenção Psicossocial (RAPS), que deve circundar o apoio institucional dos serviços de saúde mental, que, por sua vez, deve atuar como espaço de produção ativa da educação permanente e intervir na cultura manicomial presente, tanto nas práticas de cuidado operadas na atenção básica, quanto na forma da comunidade relacionar-se com os sujeitos em sofrimento psíquico (NUNES; LANDIM, 2016, p.349).

Além disso, dentro do Ministério da Saúde, há a Secretaria de Gestão do Trabalho e da Educação em Saúde (SGTES), criada em 2003, para direcionar os recursos humanos como foco na dimensão do trabalho e da educação na saúde de maneira estratégica. Para tanto, a SGTES oferta "políticas orientadoras de gestão, formação, qualificação e regulação dos trabalhadores da saúde no Brasil”, sendo que uma delas é a Política Nacional de Educação Permanente em Saúde (PNEPS). (OLIVEIRA, 2009, p.13) Esta busca colaborar para a formação e desenvolvimento dos trabalhadores da saúde. Ainda, como ação da SGTES, há o Programa de Capacitação Gerencial para o Sistema Único de Saúde, direcionado à qualificação de gestores e frentes de saúde. Portanto, as políticas e programas existem, há a preocupação com a formação continuada no profissional, contudo, precisam ser procurados para implementação ou utilização pelas equipes e gestores.

\section{CONSIDERAÇÕES FINAIS}

Conclui-se que a prática de condução de grupos com os usuários dos Centros de Atenção Psicossocial (CAPS) mostra-se como uma tarefa inquietante para o profissional, pois o suscita a sempre buscar a flexibilidade e o aprimoramento teórico e técnico. Entende-se que o planejamento e organização caracterizam-se como importantes características para a viabilização de um serviço de qualidade, contudo considera-se que, para ser possível que o trabalho seja realizado, deve-se levar em conta que o profissional lida com seres humanos e, como tal, cada pessoa tem seu momento e suas demandas para serem trabalhadas (e que influenciarão na maneira com que irão lidar com seus tratamentos).

Percebeu-se ainda, pela autoavaliação dos profissionais dos CAPS investigados, como a intervenção em grupo é gratificante e lhes possibilita o sentimento de serem úteis aos usuários e familiares. A satisfação em relação a esta modalidade de intervenção foi predominante, indicando como é necessário que haja entre os profissionais uma identificação com o que estão realizando para que possa ser constatado o comprometimento e dedicação à comunidade. Tais características mostram-se indispensável para o desenvolvimento e oferta de intervenções de qualidade e eficazes.

Já referente à Educação Permanente, pode-se identificar como se torna indispensável ao profissional manter-se atualizado e em constante busca pelo aprimoramento teórico-técnico que possibilite que suas intervenções sejam qualificadas. 


\section{REFERÊNCIAS}

ALVERGA, A. R.; DIMENSTEIN, M. Psychiatric reform and the challenges posed by deinstitutionalization. Interface - Comunic., Saúde, Educ., v.10, n.20, p.299-316, jul/dez 2006.

AMÂNCIO FILHO, A. Dilemas e desafios da formação profissional em saúde. Interface - Comunic., Saúde, Educ., v. 8, n. 15, p. 375-380, mar./ago., 2004.

BARBOSA, G. C.; COSTA, T. G. da; MORENO, V. Movimento da luta Antimanicomial: trajetória, avanços e desafios. Cad. Bras. Saúde Mental, Rio de Janeiro, v. 4, n. 8, p. 45-50, jan./jun. 2012.

BARDIN, L. Análise de conteúdo. Lisboa: Edições 70. 2000.

BRASIL. Lei n. 10.216, de 06 de abril de 2001. Dispõe sobre a proteção e os direitos das pessoas portadoras de transtornos mentais e redireciona o modelo assistencial em saúde mental. Diário Oficial da União, Brasília, DF, 2001.

Portaria MS n. 336, de 19 de fevereiro de 2002. Define e estabelece diretrizes para o funcionamento dos Centros de Atenção Psicossocial. Diário Oficial da União, Brasília, DF, 2002.

Ministério da Saúde. Secretaria de Atenção à Saúde. Departamento de Ações Programáticas e Estratégicas. Saúde mental no SUS: os centros de atenção psicossocial. Brasília: Ministério da Saúde, 2004.

Ministério da Saúde. Secretaria de Atenção à Saúde. Departamento de Atenção Básica. Cadernos de Atenção Básica: saúde mental, n. 34. Brasília: Ministério da Saúde, 2013.

CASTANHO, P. Uma Introdução aos Grupos Operativos: Teoria e Técnica. Revista do NESME, v. 9, n. 1, p. 47-60, 2012.

FREUD, S. A Ansiedade In: Conferências Introdutórias sobre a Psicanálise (Parte III) (1915-1916), v. XVI, p. 393-412.Rio de Janeiro: Imago Editora, 1996.

GONÇALVES, A. M.; SENA, R. A Reforma Psiquiátrica no Brasil: contextualização e reflexos sobre o cuidado com o doente mental na família. Revista Latino de Enfermagem. Ribeirão: v. 09, n. 2, p. 48-55, 2001.

LIMA, L. et al. Satisfação e insatisfação no trabalho de profissionais de saúde da Atenção Básica. Esc Anna Nery, v. 18, n. 1, p. 17-24, 2014.

LLAPA-RODRÍGUEZ, E. O. et al. Comprometimento organizacional e profissional da equipe de saúde. Enfermaría Global. n. 17, p. 1-16, out. 2009.

MINAYO, M. C. S. O desafio do conhecimento: pesquisa qualitativa em saúde. 7 ed. Rio de Janeiro: Abrasco, 2000.

NUNES, M.; LANDIM, F. L. P., (Org.). Saúde mental na atenção básica: política e cotidiano. Salvador: EDUFBA, 2016.

OLIVEIRA, R. G. (Org.). Qualificação de gestores do SUS. Rio de Janeiro: EAD/Ensp, 2009.

PAULA, P. P. Saúde mental na Atenção Básica: política, trabalho e subjetividade. 2011. 202 p. Tese (Doutorado em Psicologia Social) - Instituto de Psicologia, Universidade de São Paulo, São Paulo. 2011.

RICHARDSON, R. J. Pesquisa social: métodos e técnicas. 3 ed. São Paulo: Atlas, 
2008.

ROSSONI, E.; LAMPERT, J. Formação de profissionais para o Sistema Único de Saúde e as diretrizes curriculares. Boletim de Saúde. Porto Alegre, v. 18, n. 1, p. 87-98, jan./jun. 2004.

SANTOS, E. G. dos. O grupo como estratégia terapêutica nos Centros de Atenção Psicossocial Álcool e Drogas do Espírito Santo. 2010. 125 f. Dissertação (Mestrado em Saúde Coletiva) - Centro de Ciências da Saúde, Universidade Federal do Espírito Santo, Vitória, 2010.

SILVA, A. L. A. C. et al. Atividades grupais em saúde coletiva: características, possibilidades e limites. Revista de Enfermagem da UERJ, v. 11, p. 18-24, 2003.

SOUZA, A. C. de.; VASCONCELLOS, M. M.; AMARANTE, P. Novas perspectivas em atenção psicossocial. Dynamis Revista Tecno-Científica. FURB, Blumenau-SC, v.12, n. 46, p. 23-28, jan./mar. 2004.

TENÓRIO, F. A Reforma Psiquiátrica brasileira, da década de 1980 aos dias atuais: história e conceito. Hist., Cienc., Saude - Manguinhos, v.9, n.1, p.25-59, 2002. 